\title{
Finite element concept to derive isostatic residual maps - Application to Gorda Plate and Sierra Nevada regions
}

\author{
K MALLICK* and K K SHARMA** \\ *National Geophysical Research Institute, Hyderabad, India. \\ **Department of Appl. Geology; University of Madras, Chennai, India.
}

\begin{abstract}
A new space-domain operator based on the shape function concept of finite element analysis has been developed to derive the residual maps of the Gorda Plate of western United States. The technique does not require explicit assumptions on isostatic models. Besides delineating the Gorda Plate boundary, the residual maps exhibit a close match both in their anomaly patterns and magnitudes with previously computed residual maps based on the theory of isostasy.
\end{abstract}

\section{Introduction}

The tectonically active regions of the world are invariably associated with extreme topographic relief, for example the Alpine-Himalayan belt, the western United States and the south American mountain range along the west coast. The high topography according to the principle of isostasy is supported by the deepening and thickening of the low-density crustal material causing deep-seated mass deficiencies. These produce large regional lows that override or tend to mask the smaller anomalies originating from the mid and upper crustal geologic structures. The interpretation and understanding of these structures depend on how effectively the regional gravity anomalies are isolated so as to construct the isostatic residual maps.

Very accurate geophysical studies have been carried out to establish the nature of isostatic compensation in the western United States, specially in the coastal regions and Sierra Nevada. The effect of isostasy has been estimated for this region by more than one technique (Oliver 1977; Jachens and Griscom 1985). Based on the least-squares analysis (Oliver 1977), the regional Bouguer anomaly, $g_{d}$, where $d$ refers to deeper structures, was best approximated in this area by a relation, $g_{d}=-0.965 E_{64}+2 \mathrm{mGal}$. The elevation in meters, $E_{64}$, is averaged to a radius of $64 \mathrm{~km}$ of the terrain surrounding the gravity station. The computa- tions were also carried out for radius, $r=8,16,32$ and $128 \mathrm{~km}$. However, the regional field corresponding to the radius of $64 \mathrm{~km}$ appeared to be the most accepted one in Sierra Nevada. As it appears, the empirical relation to compute the regional Bouguer anomaly must be established specifically for a given region. But it is not always possible to compute regional Bouguer anomaly at a large number of stations. It was, therefore, felt necessary to develop a technique that can simplify the computational procedure. One such technique was put to practice in the early 1980s. In an interesting paper (Jachens and Griscom 1985), the regional anomalies for the western United States were computed by assuming a physical isostatic model in which the physical parameters (density of the topography $\rho$; the normal crustal thickness (at the sea level), $T$; and the density contrast at the base of the crust, $\Delta \rho)$ are estimated by extensive field measurements and seismic refraction surveys. Figure 1 shows a simplified Airy-Heiskanen isostatic-compensation model of Jachens and Griscom (1985) for the western United States and the prescribed parameters are, $\rho=2.67 \mathrm{~g} / \mathrm{cm}^{3}, T=25 \mathrm{~km}$ and $\Delta \rho=0.4 \mathrm{~g} / \mathrm{cm}^{3}$. This is the standard model.

Since these parameters are likely to vary from place to place, an upper bound and a lower bound were fixed for the regional anomaly by taking three sets of model parameters, $\rho=2.50,2.67,2.85 \mathrm{~g} / \mathrm{cm}^{3} ; T=20$, $25,30 \mathrm{~km}$, and $\Delta \rho=0.2,0.4,0.6 . \mathrm{g} / \mathrm{cm}^{3}$. The crustal

Keywords. Finite element concept; isostatic anomaly; Gorda Plate; Sierra Nevada. 


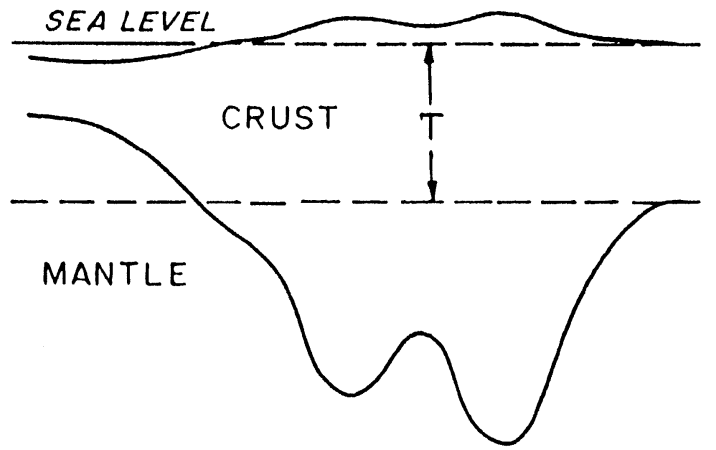

Figure 1. The Airy-Hciskanen isostatic model for western United States. For the standard model, crustal thickness, $T=25 \mathrm{~km}$; the crustal density $\rho=2.67 \mathrm{~g} / \mathrm{cm}^{3}$, and the density contrast at the base of the crust $\Delta \rho=0.4 \mathrm{~g} / \mathrm{cm}^{3}$ (after Jachens and Griscom 1985).

thickness varies between $\pm 5 \%$ from the standard anomaly. The regional anomalies computed by the above procedure were subtracted from the observed Bouguer gravity values to obtain the isostatic residual map. This technique is straight-forward and is based on a physical model.

The two methods described above depend on the isostatic model assumed to represent the crustal structure in the western United States. It is likely that the isostatic condition will vary from region to region. In other words, the empirical relation (Oliver 1977) and the isostatic model (Jachen and Griscom 1985) that are valid for western United States cannot be directly employed elsewhere to compute the isostatic residual maps. Chapin (1996), for instance, has estimated $\rho=$ $2.60 \mathrm{~g} / \mathrm{cm}^{3}, T=30 \mathrm{~km}$ and $\Delta \rho=0.45 \mathrm{~g} / \mathrm{cm}^{3}$ to compute the isostatic residual map of South America. The desirability to develop a technique that is universally valid, provided the motivation for the approach discussed here. It is based on finite element analysis (FEA) in a manner that is free from the need to assume an empirical relation or a physical model. It also does away with the need to compute the regional anomalies related to topography and isostasy. Furthermore, it does not call for the assumption of uniform crustal density over large regions. This information is implicit in the Bouguer gravity anomaly. Finally, this approach is not area-specific. The elimination of the first three steps to compute regional and residual anomalies makes the FEA approach a unique one.

In the present scheme, the region of interest in the Bouguer gravity map is superimposed by a network of finite elements (Mallick and Sharma 1997, 1999). The gravity values at the nodes of the finite element are assumed to represent the regional, which are interpolated into the map space with the help of the shape functions of the element (Cheung and Yea 1979). The shape functions are, in fact, the weighting functions. These are defined without ambiguity.

We have illustrated the application of this new concept to two areas, namely, the Gorda Plate sub- duction zone and the Sierra Nevada of California for the following reasons. First, we can compare the regional anomalies and the FEA residual maps of these regions with previously obtained accurate regional anomalies and isostatic residual maps (Oliver 1977; Jachens and Griscom 1985). Second, these two regions represent two different geological environments; the Gorda Plate lies near the coast and is a subduction zone, whereas the Sierra Nevada granitic batholith lies inland.

\section{The new method}

We outline below the principle and the computational procedure of the FEA technique. Figure 2(a) shows the Bouguer gravity map of northern California. We have separately superimposed on it two elements, one on the north, marked I, consisting of the Klamath Mountains and the Cascade Range (Jachens and Griscom 1985; Blakely 1995) and another, marked II, covering the Gorda Plate region. Filled circles on these elements indicate eight nodes; and the gravity values at these nodes are assumed to represent the regional gravity values. These two elements in the real map space are defined by $x-y$ cartesian co-ordinates. The computations can however be simplified, if the real $x-y$ space is substituted by a reference $\xi-\eta$ space, $\xi$ and $\eta$ are non-dimensional and vary between -1 and 1 . The reference space in figure 2(b) can represent both the above elements. The full circles are the nodes. The regional Bouguer anomaly $g(\xi, \eta)$ is expressed as:

$$
\left.g_{d}(\xi \eta)=\sum N_{i} \xi, \eta\right) g_{i} \quad i=1,2, \ldots, 8
$$

where $g_{i}$ stand for the gravity values at the nodes of the element, and $N_{i}(\xi, \eta)$ are the shape or weighting functions (Cheung and Yeo 1979). Equation (1) is valid for both the regions I and II, although these have different dimensions and different orientations. The weighting functions corresponding to any point $(\xi, \eta)$, too, are the same for both these regions. The gravity values at the nodes of the two elements are, however, different.

The regional Bouguer anomaly computed grid-wise or at points distributed at random are transferred to the $x-y$ map space by a co-ordinate transformation given by

$$
\begin{aligned}
& x(\xi, \eta)=\sum N_{i}(\xi, \eta) x_{i}, \\
& y(\xi, \eta)=\sum N_{i}(\xi, \eta) y_{i},
\end{aligned}
$$

where $x_{i}$ and $y_{i}$ are the local co-ordinates corresponding to the elements I and II. The shape functions or the weighting functions in equations (1) and (2) are identical. The elements with these properties are called isoparametric. The co-ordinate transformations can also be carried out by weighting functions that may be different from those used in equation (1). It may be 

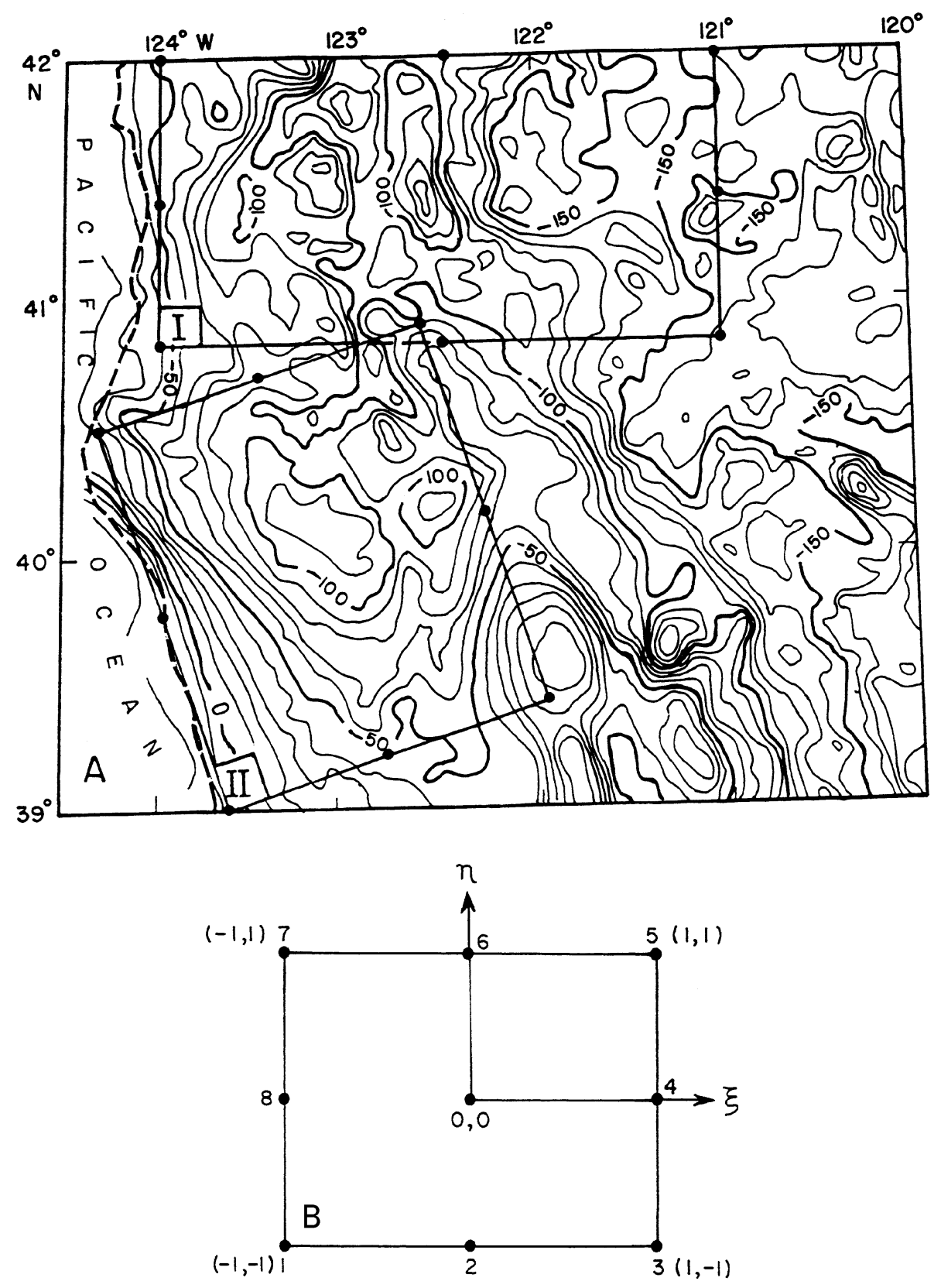

Figure 2(A). Bouguer gravity of parts of northern California. The Klamath Mountain and Cascade Range are superimposed by an element marked I and the Gorda Plate and Sierra Nevada batholith by another element marked II. Each element has eight nodes shown in full circles. The profile at $41^{\circ} \mathrm{N}$ (figure 3) lies in element I. Contour interval: 10mGal. (B). A reference element in $\xi-\eta$ space corresponding to the above elements in $x-y$ space is obtained by a simple substitution, $\xi=\left(x-x_{c}\right) / a$ and $\eta=\left(y-y_{c}\right) / b$, where $x_{c}, y_{c}$ define the centre of the element, and 2(A) and 2(B) are the sides of the element. The non-dimensional co-ordinates $\xi$ and $\eta$ vary between -1 and 1 .

noted that figure 3 relates to eight-node isoparametric quadratic elements. One can as well choose twelvenode cubic elements or any mixed elements. Accordingly, the shape functions need to be defined.

\subsection{Accuracy}

A test of accuracy is essential for any numerical technique. For this new method, we provide two cases to verify the accuracy. First, we have recomputed by our FEA method (Sharma et al 1999, figure 1) the regional and residual components of a synthetic model consisting of a spherical target in the presence of a regional trend due to a dipping plane earlier used by Agocs (1951) to demonstrate the application of least squares (LS) technique to compute the regional and residual anomalies. We summarize the results in table 1 that shows the comparison of the model, least squares (LS) and FEA regional anomalies.

The finite element residual anomaly in the table shows a departure of just 0.1 units $(0.28 \%)$, whereas the least squares residual value has a departure 2.5 
Table 1. Comparison of the model, LS and the FEA regional and residual anomalies.

\begin{tabular}{|c|c|c|c|}
\hline Method & Regional & $\begin{array}{l}\text { Residual } \\
\text { (peak } \\
\text { response } \\
\text { of the } \\
\text { sphere) }\end{array}$ & $\begin{array}{c}\text { Difference } \\
\text { from the } \\
\text { model } \\
\text { response }\end{array}$ \\
\hline The model & $g_{d}($ model $)=20 y+40$ & 34.8 & 0 \\
\hline Agocs (1951) & $=20.03 y+42.29$ & $9 \quad 32.3$ & -2.5 \\
\hline Present study & $g_{d}(\mathrm{FEA})=20 y+40$ & 34.9 & 0.1 \\
\hline
\end{tabular}

units $(7.18 \%)$. Second, the accuracy of the method is tested by computing the regional along the east-west profile at $41^{\circ} \mathrm{N}$ and comparing it with the previously computed regional (Jachens and Griscom 1985, figure $5)$. The open circles in figure 3 represent the FEA regional. Except for one value in the coastal region, it is remarkable that the FEA regional and the regional corresponding to the standard isostatic model match closely; the average difference is $1 \mathrm{mGal}$. The inset in figure 3 shows the Bouguer gravity map, FEA and isostatic residual profiles along $41^{\circ} \mathrm{N}$.

The experiment assumes considerable relevance from the standpoint that the FEA approach yields regional and residual anomalies as satisfactory as those obtained by approximating a physical model. This lifts the FEA approach from a local to the global context in the sense that it can be used in any part of the world even without a fair knowledge of the isostatic conditions prevailing in that region.

\section{Field applications}

\subsection{Gorda Plate}

North of the Cape Mendocino in northern California between the coast and the Coast Range Thrust there exists a gravity low. This low is terminated on the south by a narrow zone marked by a dash-dot line in figure 2(a). There is a change in gradient in gravity contours on both sides of the dash-dot line, and more so on the south. This gravity low manifests the presence at depth of the subducted Gorda Plate, the southern edge of which is reflected in the magnetic data and the distribution of seismicity. The residual gravity map of the Gorda Plate region prepared by the FEA approach and the isostatic residual map prepared by assuming a physical model based on the theory of isostasy are illustrated in figures 4(a) and (b) respectively. These two maps are similar in pattern and the order of the magnitudes of the anomaly is the same.

In the next example, we consider the Sierra Nevada region (Oliver 1977) away from the coast. One profile

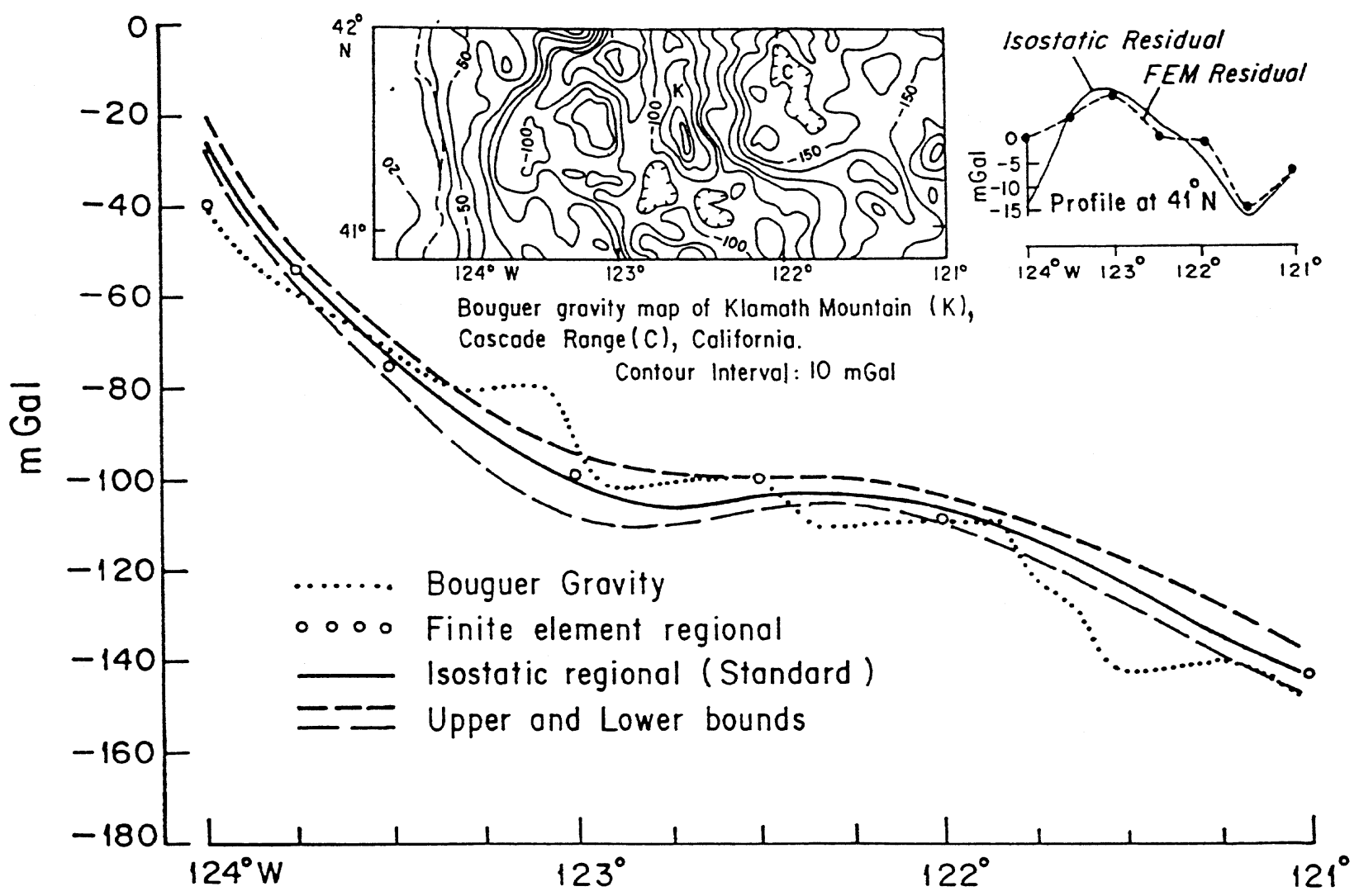

Figure 3. The regional and gravity field along an east-west profile at $41^{\circ} \mathrm{N}$ based on the model shown in figure 1 . The standard curve in continuous line lies between an upper and lower bound. The FEA regional anomaly is shown by open circles. 

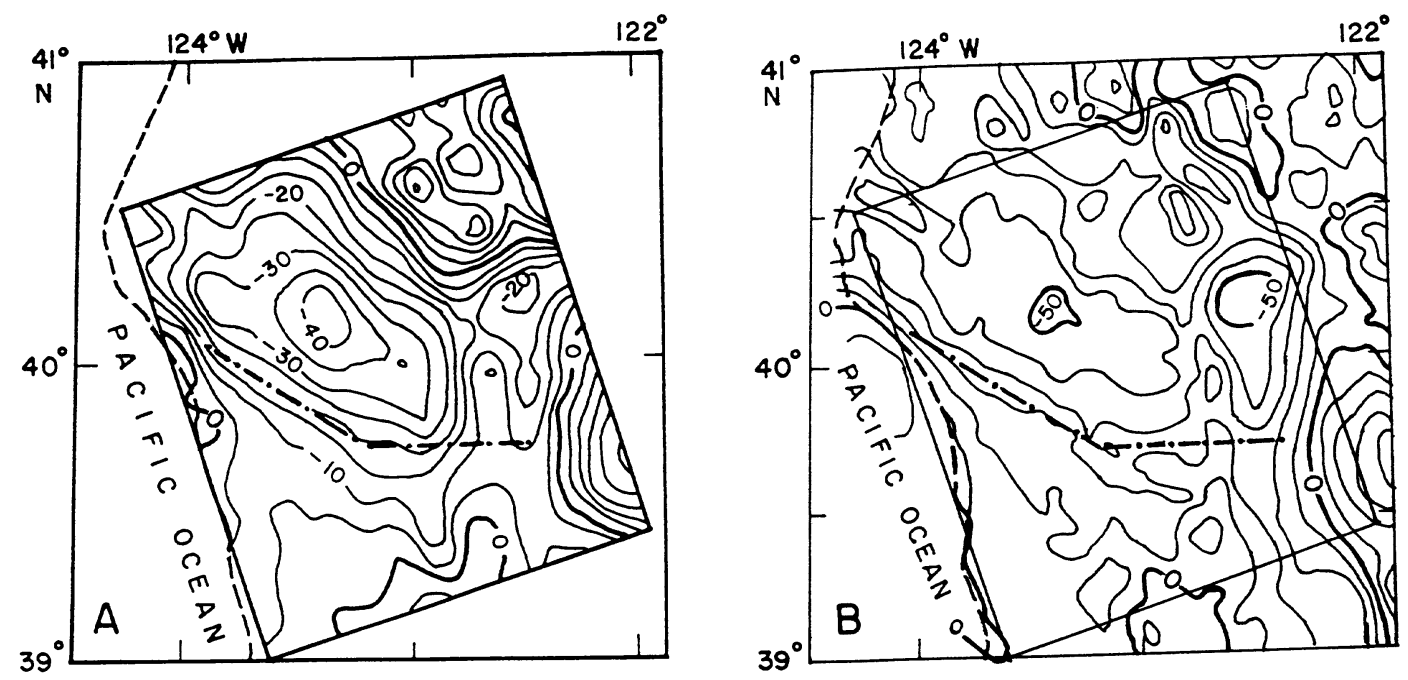

Figure 4(A). The residual gravity derived by FEA approach and (B) the isostatic residual map obtained by approximating the isostatic-compensation model of the Gorda Plate subduction zone. Contour interval: 5 mGal.

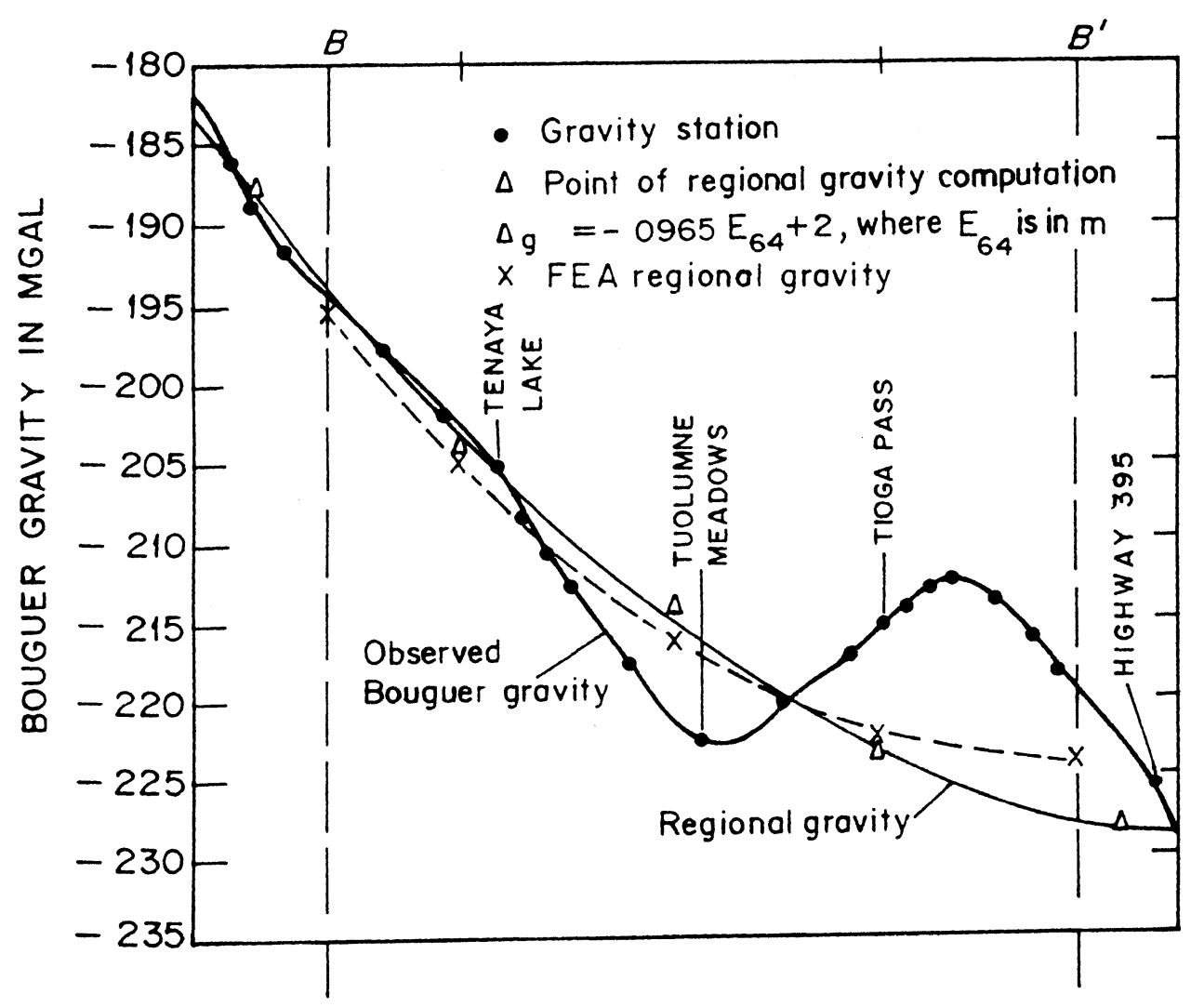

Figure 5. Bouguer gravity and regional gravity $(\Delta)$ obtained by trend surface and least-square analysis along profile BB' in eastcentral Yosemite National Park, Sierra Nevada. The continuous line is obtained by regression analysis. The broken line shows the FEA regional gravity.

in this region shows a regional gravity curve that is, in essence, an isostatic correction (Oliver 1977, profile $\mathrm{BB}^{\prime}$ in figure 5) with respect to the data along this particular profile. We have computed the FEA regional anomaly along the same profile and drawn a comparison in figure 5. Out of five stations two end stations fall outside the range of $\mathrm{BB}^{\prime}$ profile, thereby leaving just three points for comparison. The match between the two regional Bouguer curves is once again remarkable. The discrepancy on the right end may be due to the use of gravity value at station Highway 395. We have on the other hand used the gravity value coinciding $\mathrm{B}^{\prime}$ as per the gravity map of Oliver (1977, figure 5). 


\section{Discussion}

The examples cited in this paper show that the FEA residual maps compare rather well with those obtained on the assumption of an empirical relation and a physical isostatic model. In the absence of adequate field studies including magnetics and seismic refraction surveys, it is not feasible to assign physical parameters to an isostatic model. In such situations, we believe that the FEA concept provides a more objective representation of the regional anomalies and residual maps that can be used with greater confidence to interpret the subsurface geology, in particular, the mid and upper crustal geological structure. The FEA approach does not claim to replace all the earlier technologies; its aim is to strengthen and supplement the existing techniques.

\section{Acknowledgements}

The authors wish to thank Dr. H K Gupta, Director, National Geophysical Research Institute, Hyderabad for his interest in this work and Mr. Ch Venkateswarlu and Mr. G Ramakrishna Rao for their help in computations and preparation of the manuscript.
The authors had very useful discussions with Dr. A Roy.

\section{References}

Blakely R J 1995 Potential theory in gravity and magnetic applications (Cambridge Univ. Press).

Chapin D 1996 A deterministic approach toward isostatic gravity residuals - A case study from South America; Geophysics 61 1022-1033

Cheung Y K and Yeo M F 1979 A practical introduction to finite element analysis (Pitman).

Jachens R C and Griscom A 1985 An isostatic anomaly map of California - A residual map for interpretation of anomalies from infracrustal sources: In The utility of regional gravity and magnetic anomaly maps (ed) W J Hinze (SEG) 347-360.

Mallick K and Sharma K K 1997 Computation of regional gravity anomaly - A novel approach; Proc. Indian Acad. Sci. (Earth Planet Sci.) 106 55-59

Mallick K and Sharma K K 1999 A finite element method for computation of the regional gravity anomaly; Geophysics $\mathbf{6 4}$ 461-469

Oliver H W 1977 Gravity and magnetic investigations of the Sierra Nevada batholith; Geol. Soc. Amer. Bull. 88 445-461

Sharma K K, Rao V K and Mallick K 1999 Finite element gravity regional and residual anomalies and structural fabrics of northwest Ganga basin; J Geol. Soc. India $\mathbf{5 4}$ 169-178 